\title{
Neural Network Optimized Model Predictive Multi-Objective Adaptive Cruise Control
}

\author{
Siyi Zhang ${ }^{1}$, Junzhi Zhang ${ }^{1,2}$ \\ ${ }^{1}$ State Key Laboratory of Automotive Safety and Energy, Tsinghua University, Beijing, People's Republic of China \\ ${ }^{2}$ Innovation Center of Electric Vehicles, Beijing, People's Republic of China
}

\begin{abstract}
A model predictive multi-objective adaptive cruise control (MPC MO-ACC) system, designed to consider both the tracking performance and the fuel consumption, is optimized by a neural network in this paper, reducing the computational complexity without sacrificing the control performance. The optimized MO-ACC control system is built by training a neural network with the control results of the MPC MO-ACC system. Simulation tests are conducted in Matlab/Simulink in conjunction with the high-fidelity CarMaker software. Influences of four driving conditions (the learning track, NEDC, JP05, FTP75) and two kinds of sensor models (ideal radar sensor and 77GHz physical radar sensor) are analysed. Simulation results have shown that the neural network optimized model predictive MO-ACC has the same control capability and strong robustness as the original MPC MO-ACC. Meanwhile, the optimized control system has much lower computational complexity, which shows potentials for the application in real-time vehicle control and industry.
\end{abstract}

\section{Introduction}

With the progress of intelligent vehicle control, the normal Adaptive Cruise Control (ACC) system, which has the basic functions such as cruise control and active collision avoidance ${ }^{[1]}$, has developed into the Multiobjective Adaptive Cruise Control (MO-ACC) system, which considers not only the tracking performance but also the fuel consumption, and driver's comfort as well ${ }^{[2]}$.

Reference [2] developed an MO-ACC system based on Model Predictive Control (MPC). It is proved that MPC can make multi-objective optimization simultaneously, and has the features of good control performance and high robustness. However, the high computational complexity of MPC has restrained its application in real-time vehicle control.

There are two main solutions to solve this problem: 1 . improving the hardware computational ability, but will increase costs as well; 2. building an off-line look-up table, but workload of building such a table is very high.

Meanwhile, neural network has been implemented in ACC systems. Reference [3] has built a neural network ACC system on the base of human drivers' control results provided by NGSIM, which can ensure a safe headway for vehicles under most circumstances. However, fuel consumption was not considered in such kind of neural network ACC systems.

This paper has shown an optimization of the MPC MO-ACC system by using the neural network. The optimized MO-ACC system is built by training a neural network with the control results of the MPC MO-ACC system, reducing the computational complexity without sacrificing the control performance. Compared to the previous methods, this optimization is more cost-efficient and labour-efficient.

This paper is organized as follows. In Section 2, the training process of the network is introduced. In Section 3, results of the simulation conducted in Matlab/Simulink in conjunction with the high-fidelity CarMaker are analysed. The influences of different driving conditions and the noise of physical radar sensors are also considered in the analysis. In Section 4, the advantages of this neural network optimized model predictive multi-objective adaptive cruise control system are concluded.

\section{Training of the neural network}

The neural network is trained with the control results of the MPC MO-ACC, when ideal sensors are used. The vehicle speed, acceleration and headway of the preceding and following vehicles, and the acceleration command value of the previous step are selected as inputs of the control system, then the acceleration command value of this step is calculated as the output.

Series of experiments are done to compare the influences of different network structures (fitnet, cascadeforwardnet and feedforwardnet), network nodes (10, 15 and 20 nodes), activation functions (tansig, puelin) and training functions (taingd, traingdm, traingdx, trainrp, traincgf, traincgp, traincgb, trainscg, trainbfg, trainoss, trainlm, trainbr) on the control performance. Each trial 
was repeated 5-10 times to ensure the reliability of the results.

It is shown that with the network structure of multilayer feedforward neural network, one hidden layer, ten nodes, activation function 'tansig' for hidden layer, activation function 'puelin' for output layer, and training function 'trainlm' or 'trainbr', neural network control systems with good performance can be trained. The settings of the neural network used in this paper are as shown in Table 1.

Table 1. Settings of the neural network control system.

\begin{tabular}{|c|c|}
\hline Network Element & Setting \\
\hline Network structure & $\begin{array}{c}\text { Multi-layer feedforward neural } \\
\text { network }\end{array}$ \\
\hline $\begin{array}{c}\text { Number of hidden } \\
\text { layers }\end{array}$ & 1 \\
\hline Number of nodes & 10 \\
\hline $\begin{array}{c}\text { Activation function } \\
\text { of hidden layer }\end{array}$ & tansig \\
\hline $\begin{array}{c}\text { Activation function } \\
\text { of output layer }\end{array}$ & puelin \\
\hline Training function & trainlm \\
\hline
\end{tabular}

\section{Co-simulations and analysis}

Co-simulations are conducted in MATLAB/Simulink and IPG CarMaker to compare the control performance of the original MPC and the neural network control system. The vehicle data of Honda Fit CVT provided by CarMaker 6.0.2 is chosen to build the vehicle dynamic model. An ideal radar sensor and a $77 \mathrm{GHz}$ physical radar sensor in CarMaker are used respectively to detect the relative distance, relative vehicle speed, and relative longitudinal acceleration. Together with the motion signals of the following vehicle, these signals are then calculated to get the inputs of the MO-ACC systems.

The MPC and neural network MO-ACC systems are evaluated by the tracking performance and the fuel consumption. To reflect tracking capability, the tracking error index (TEI) similar to that was defined in Reference [4] is used. It considers both speed error and distance error, as shown in Equation (1).

$$
T E I=\frac{1}{T} \int_{0}^{T}(|\Delta d(\mathrm{t})|+0.1|\Delta v(\mathrm{t})|) d t
$$

Where $T$ is the simulation time, $\Delta d$ is the difference between the desired distance and the actual distance in every step. $\Delta v$ is the difference between the desired and actual velocity in every step. The units of $\Delta d$ and $\Delta v$ are $\mathrm{m}$ and $\mathrm{m} / \mathrm{s}$ respectively.

The fuel consumption is reflected by the average fuel consumption per hundred kilometres (FCM). It is calculated as Equation (2).

$$
F C M=\frac{1}{s} \int_{0}^{T} Q_{\text {eng }} d t
$$

Where $Q_{\text {eng }}$ is the fuel consumption rate of the engine, whose unit is L/s. $S$ is the total driving distance of the following vehicle, whose unit is $100 \mathrm{~km}$.

\subsection{Simulation results using ideal radar sensors}

Firstly, ideal radar sensors are used in the simulation, which means the relative velocity, relative distance and relative acceleration are very accurate, and so the control will not be influenced by the noise of sensors.

An MPC MO-ACC system is then tuned under the track shown in Figure 1. The target velocity and target headway, shown in black dashed lines, are tightly followed by the actual velocity and actual headway, which are shown in blue lines. The relative velocity between the preceding vehicle and the following vehicle is controlled within $\pm 1 \mathrm{~m} / \mathrm{s}$, and the relative distance between the target and actual headway is controlled within $\pm 0.5 \mathrm{~m}$, which can show the good performance of the MPC control system.
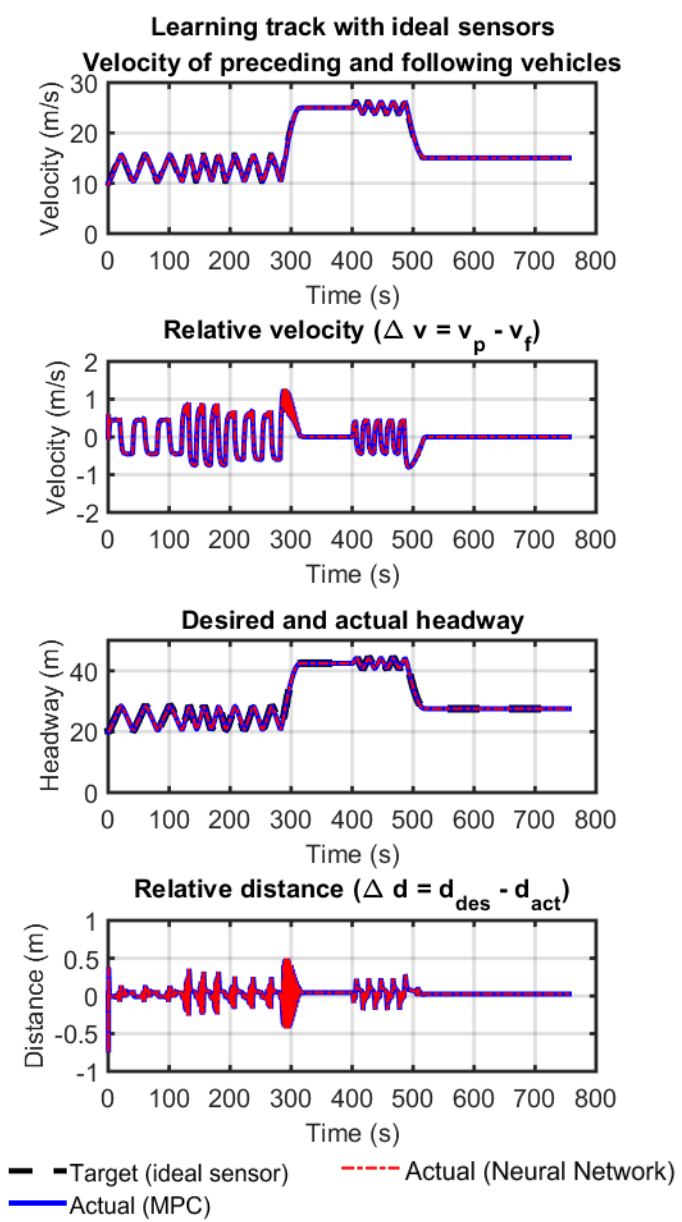

Figure 1. Simulation results under learning track with ideal sensors

After that, a neural network MO-ACC system is trained by the control results of the MPC system under the learning track with ideal sensors. The control results of this neural network MO-ACC system is shown in red lines in Figure 1, which highly overlaps that of the MPC system. This can be explained by the relative desired acceleration shown in Figure 2, which means the difference between the desired accelerations calculated 
by the MPC and the neural network control. This value is constantly within $\pm 3 \times 10^{-4} \mathrm{~m} / \mathrm{s}^{2}$, which has almost no influence in the following vehicle dynamic control.

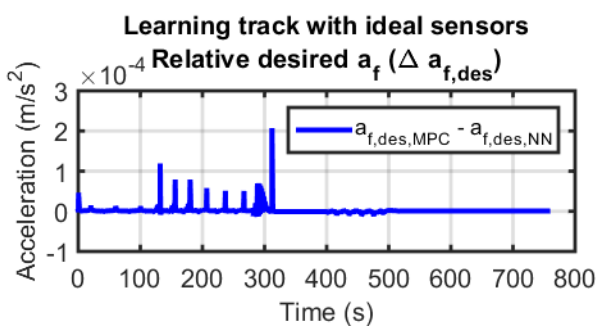

Figure 2. Relative desired acceleration under learning track with ideal sensors

Moreover, the original MPC and the neural network optimized control systems are tested under different typical worldwide driving conditions, including NEDC for Europe, JP05 for Japan, and FTP75 for the USA.
The control results shown in Figure 3 illustrate the good performance and strong robustness of both the MPC and neural network control. The relative velocity and relative distance are always controlled within $\pm 3 \mathrm{~m} / \mathrm{s}$ and $\pm 1 \mathrm{~m}$ correspondingly.

Furthermore, the tracking performance and fuel consumption of these two control systems under different driving conditions are integrated in Figure 8. The TEI and FCM values of the neural network control are totally the same as that of the MPC when using ideal sensors. This can be explained by the slight relative desired acceleration between the MPC and neural network control system, as shown in Figure 4, which is constantly within $\pm 1 \times 10^{-3} \mathrm{~m} / \mathrm{s}^{2}$. When considering the complicated dynamic control progress in the lower controller and the nonlinear characteristics of the vehicle body and tyres, it is understandable that the influence caused by such a small difference in the acceleration command is too hard to observe.
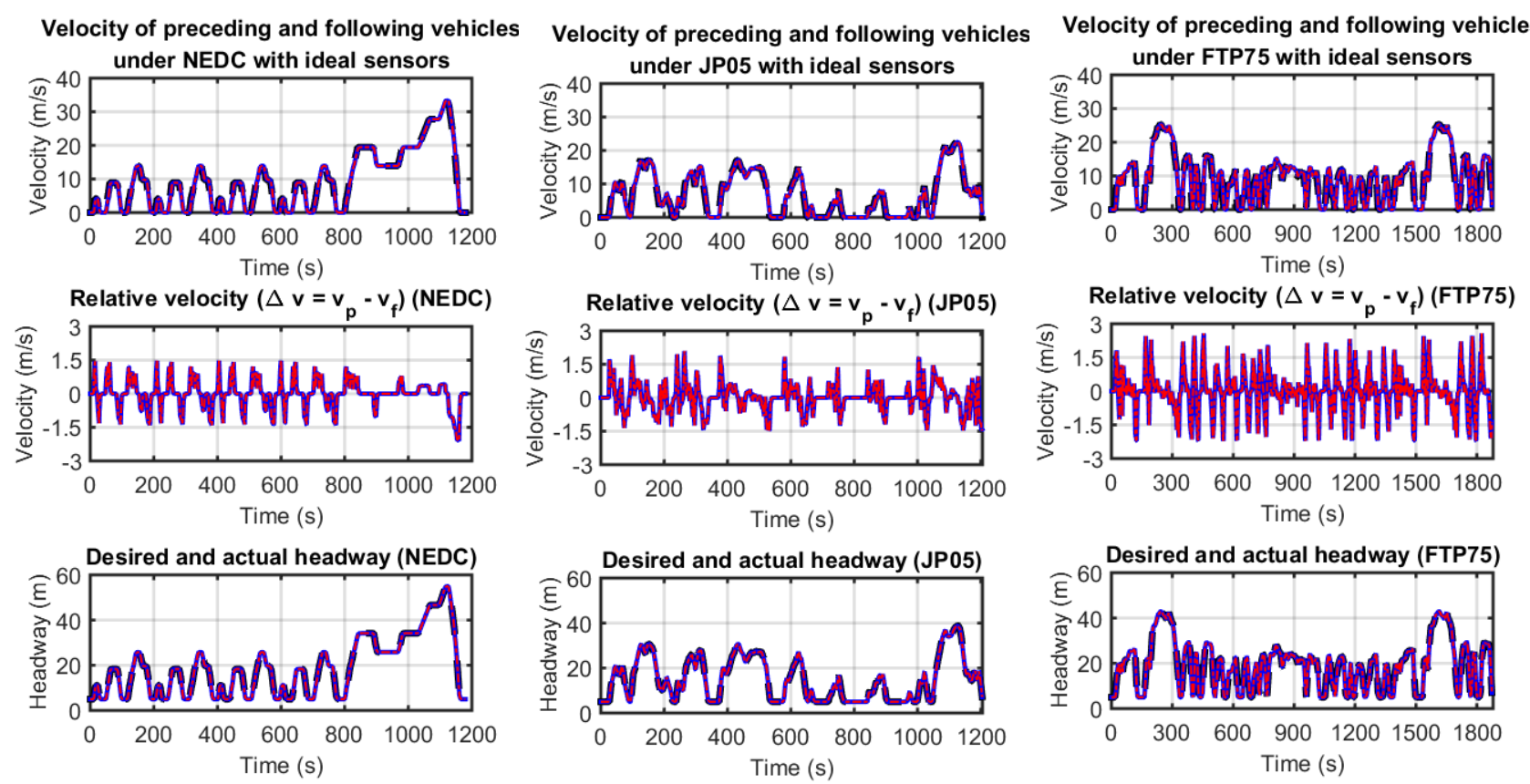

Relative distance $\left(\Delta d=d_{\text {des }}-d_{\text {act }}\right)($ NEDC)

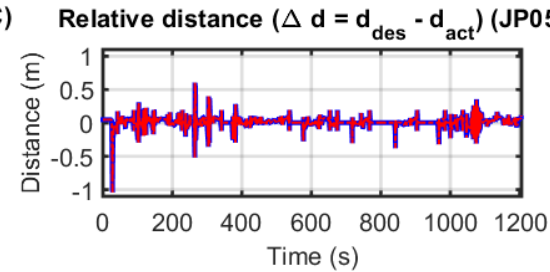

Relative distance $\left(\Delta d=d_{\text {des }}-d_{\text {act }}\right)$ (FTP75)
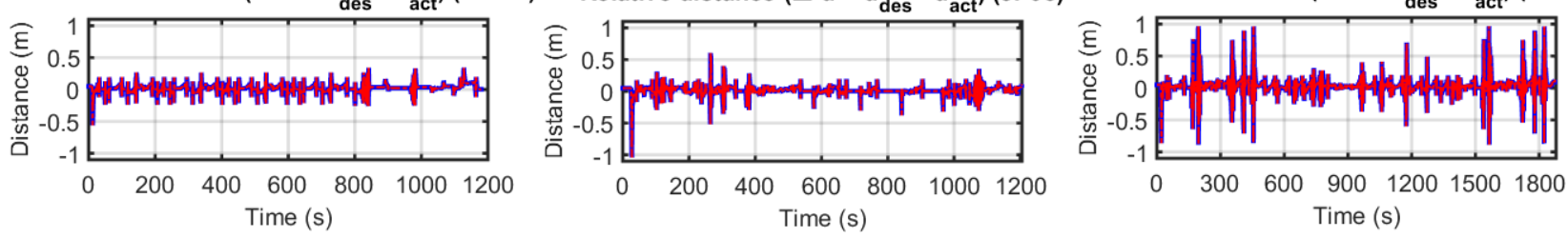

- - -Target (ideal sensor) $\longrightarrow$ Actual (MPC) -----Actual (Neural Network)

Figure 3. Simulation results under NEDC, JP05 and FTP75 with ideal sensors
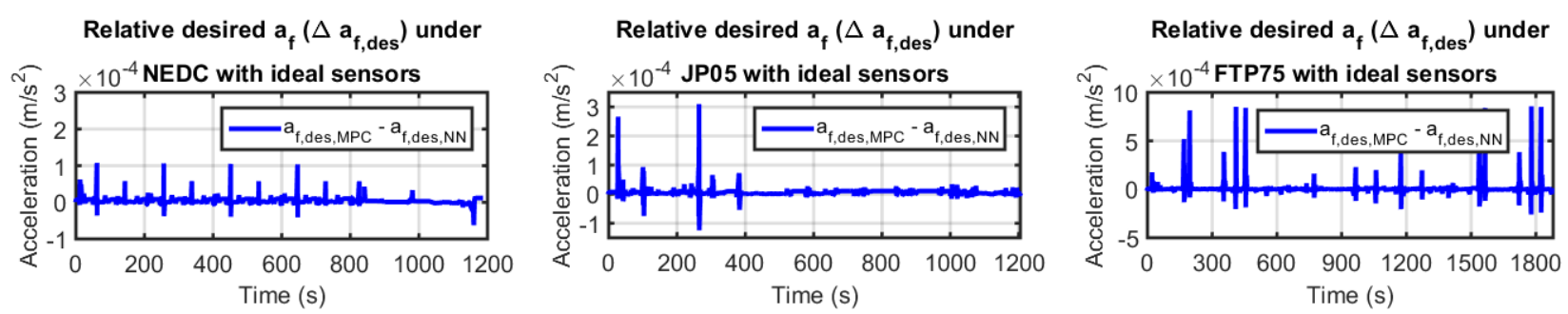

Figure 4. Relative desired acceleration under NEDC, JP05 and FTP75 with ideal sensors 
According to the tests so far, it can be concluded that, when not considering the noise of sensors, the neural network MO-ACC system has not only the same good control ability but also the high robustness as the MPC system.

\subsection{Simulation results using physical radar sensors}

In this subsection, $77 \mathrm{GHz}$ physical radar sensors are used in the co-simulation, which means the noise of sensors will be considered in the following tests. Due to the randomness of the noise, all the tests presented here are repeated for 5 times to ensure the reliability of the results. Meanwhile, TEI is still calculated by the accurate relative velocity and distance, in order to eliminate the influence of the sensor noise on these evaluation indexes.

The noise bandwidth of radar is set to be $25000 \mathrm{~Hz}$ and the noise figure is set to be $4.8 \mathrm{~dB}$, which are recommended default values in CarMaker. The influence of these noises can be seen apparently in the enlarged parts in Figure 5 and Figure 6. The relative velocity and actual distance between the preceding and following vehicles are measured by the physical sensors with noise. As a result, the velocity of the preceding vehicle and the actual headway will flutter around the accurate values given by the ideal sensors.

Even in this case, both the MPC and neural network control systems are still performing well. As shown in Figure 7 , the relative velocity and relative distance are still controlled within $\pm 1.5 \mathrm{~m} / \mathrm{s}$ and $\pm 1 \mathrm{~m}$, demonstrating the good control ability and high robustness of both control systems.

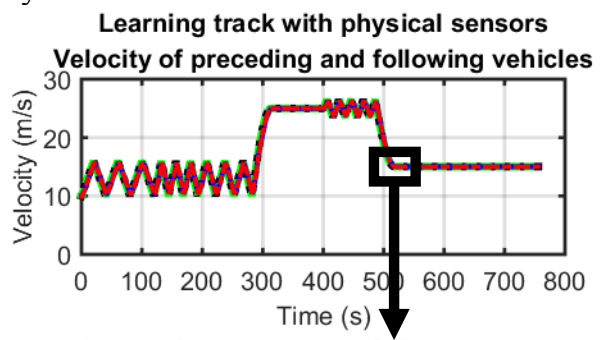

Velocity of preceding and following vehicles

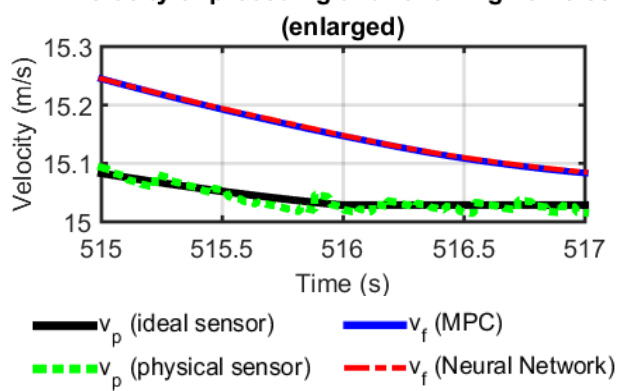

Figure 5. Velocity of preceding and following vehicles under learning track with physical sensors

However, the relative distance controlled by neural network is slightly higher than that by MPC. This can also be verified by TEI in Figure 8 . When considering the noise of sensors, TEI of the neural network control system is slightly worse than that of MPC, where relative errors are $2.07 \%$ under the learning track, $2.80 \%$ under
NEDC, 7.16\% under JC05 and 6.86\% under FTP75. However, since absolute errors are all below 0.01 , the tracking performance of the neural network MO-ACC control can still be viewed as good as that of the original MPC system.

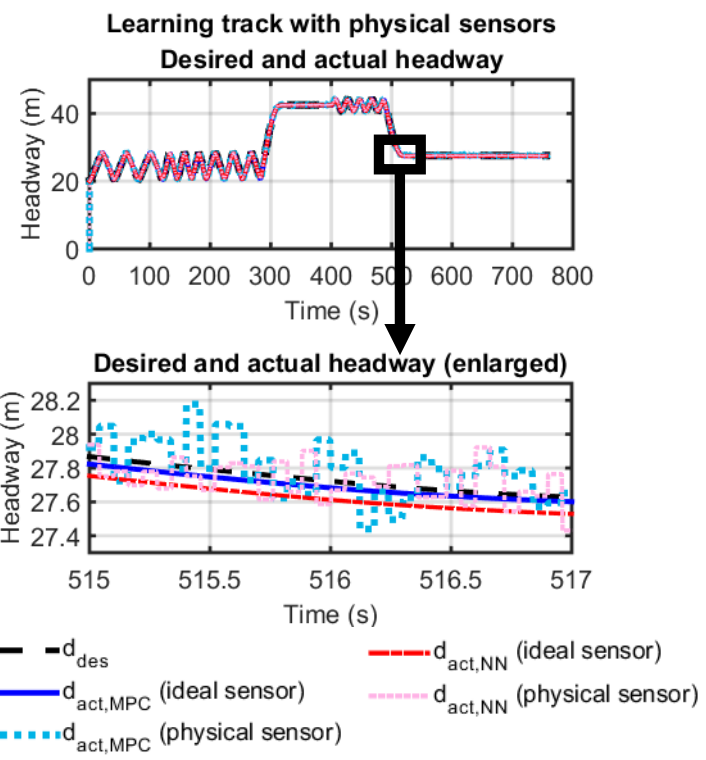

Figure 6. Desired headway and actual headway under learning track with physical sensors
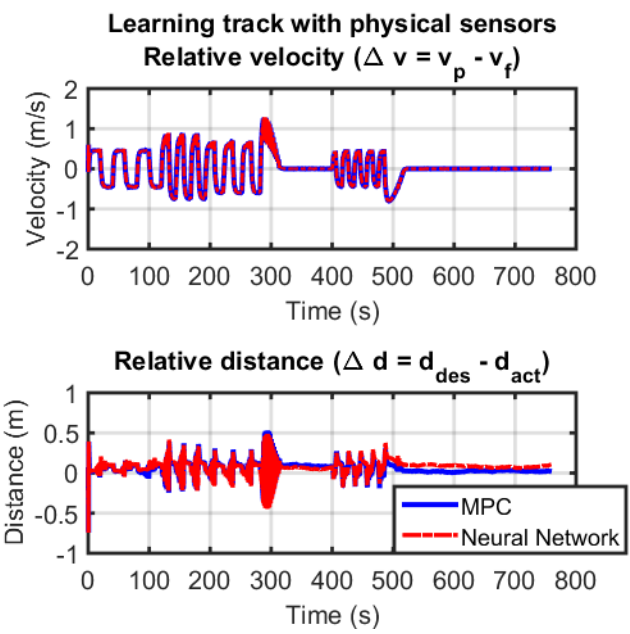

Figure 7. Relative velocity and relative distance under learning track with physical sensors

Moreover, the fuel consumption of vehicles controlled by different control systems under different driving conditions and with different kinds of sensors are shown in Figure 8. It can be seen that the influence of sensors' noise is very small, and the performance of the original MPC and the optimized neural network control system are very similar. The error between the FCM of different sensors (ideal and physical radar) and the error between the FCM of MPC and neural network control systems are all below $0.01 \mathrm{~L} / 100 \mathrm{~km}$. When tested under NEDC with physical sensors, a vehicle controlled by the neural network consumes even less fuel than a vehicle controlled by an MPC system. Thus, it can be proved that the neural network MO-ACC control system has the same control capability and strong robustness as the original 
MPC system, in aspects of both the tracking performance and fuel consumption.

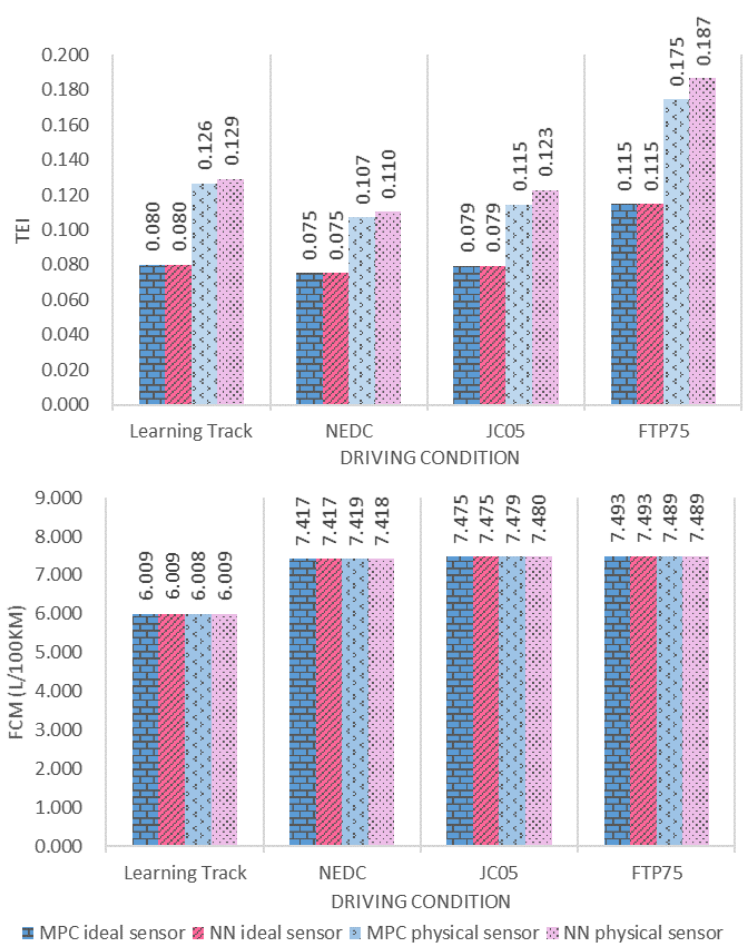

Figure 8. TEI and FCM comparison of MPC and Neural Network control systems with ideal and physical sensors

\subsection{Comparison of the computational complexity}

After comparing control capability and robustness, the computational complexity of these two control systems are analysed in this subsection, which is measured by the computing time on the same hardware. Simple lower controller and vehicle dynamic models are used here, in order to make sure that the computing time is only influenced by complexity of MO-ACC control systems.

As shown in Table 2, the computing time of the neural network control system is more than 300 times shorter than that of the MPC system. This means by using the neural network control, the computational complexity can be reduced by more than 300 times, which is a great feature in real-time vehicle control and has a great potential for the wide application in industry.

Table 2. Comparison of the computing time of the MPC system and the neural network control system

\begin{tabular}{|c|c|c|c|}
\hline & $\begin{array}{c}\text { Time using } \\
\text { MPC } \\
(\mathrm{s})\end{array}$ & $\begin{array}{c}\text { Time using } \\
\text { neural network } \\
\text { control } \\
(\mathrm{s})\end{array}$ & Multiple \\
\hline $\begin{array}{c}\text { Learning } \\
\text { track }\end{array}$ & 1200 & 4 & 300 \\
\hline NEDC & 2000 & 6 & 333 \\
\hline JP05 & 2040 & 6 & 340 \\
\hline FTP75 & 3180 & 10 & 318 \\
\hline
\end{tabular}

\section{Conclusion}

A new method to optimize model predictive MO-ACC systems is given in this paper. After being trained with the control results of the MPC MO-ACC system, the neural network MO-ACC system shows almost the same good control ability and strong robustness as the original MPC system. Meanwhile, the computational complexity of the MPC system can be reduced by more than 300 times in this optimization, which makes its application in real-time control possible.

This MPC-trained neural network MO-ACC system considers not only the tracking performance, but also the fuel consumption, which is an advantage over the normal neural network control systems trained by human drivers' control behaviour. Besides, the training process requires no big data and data cleaning. Furthermore, control systems which can consider more factors can also be designed in this method.

Moreover, compared with other methods to reduce the computational complexity of MPC, such as updating the hardware or building a large off-line look-up table, the optimization method given in this paper is more costefficient and labour-efficient. As a result, the neural network optimized model predictive MO-ACC system features not only the good control ability and strong robustness, but also the low computational complexity and low cost, which is believed to have a great potential for the application in real-time vehicle control and industry.

\section{Funding}

This work was supported by the National Key Research and Development Program of China [project no. 2016YFB0101402]; the National Natural Science Foundation of China [project no. 51475253].

\section{References}

1. J. Zhang, Z. Liu, Z. Qi, et al., Development of Automotive Adaptive Cruise Control System, Vehicle \& Power Technology 2, 44-47 (2003)

2. S. Li, J. Wang, K. Li, et al., Processing of MPC practical problems and its application to vehicular adaptive cruise control systems, J Tsinghua Univ (Sci and Tech) 50, 645-648 (2010)

3. W. Shen, The Research on Adaptive Cruise Control System Based on Neural Network and PID, Jiangsu University (2015)

4. S. Li, K. Li, R. Rajamani, et al., Model Predictive Multi-Objective Vehicular Adaptive Cruise Control, IEEE Transactions on Control Systems Technology 19, 556-566 (2011) 